\title{
Modification of the Protein Corona-Nanoparticle Complex by Physiological Factors
} Nicholas J. Braun ${ }^{a}$, Madeleine C. DeBrosse ${ }^{a}$, Saber M. Hussain ${ }^{a}$, Kristen K. Comfort ${ }^{b *}$

${ }^{a}$ Molecular Bioeffects Branch, Bioeffects Division, Human Effectiveness Directorate, 711 Human Performance Wing, Air Force Research Laboratory, Wright Patterson AFB, 2729 R. St, Bldg 837, Dayton, Ohio 45433, USA

${ }^{\mathrm{b}}$ Department of Chemical and Materials Engineering, University of Dayton, 300 College Park, Dayton, OH 45469, USA

*Corresponding author information:

E-mail: kcomfort1@udayton.edu, Telephone: +1 937-229-2627, Fax: +1 937-229-4006

\begin{abstract}
Nanoparticle (NP) effects in a biological system are driven through the formation and structure of the protein corona-NP complex, which is dynamic by nature and dependent upon factors from both the local environment and NP physicochemical parameters. To date, considerable data has been gathered regarding the structure and behavior of the protein corona in blood, plasma, and traditional cell culture medium. However, there exists a knowledge gap pertaining to the protein corona in additional biological fluids and following incubation in a dynamic environment. Using $13 \mathrm{~nm}$ gold NPs (AuNPs), functionalized with either polyethylene glycol or tannic acid, we demonstrated that both particle characteristics and the associated protein corona were altered when exposed to artificial physiological fluids and under dynamic flow. Furthermore, the magnitude of observed behavioral shifts were dependent upon AuNP surface chemistry. Lastly, we revealed that exposure to interstitial fluid produced protein corona modifications, reshaping of the nano-cellular interface, modified AuNP dosimetry, and induction of previously unseen cytotoxicity. This study highlights the need to elucidate both NP and protein corona behavior in biologically representative environments in an effort to increase accurate interpretation of data and transfer of this knowledge to efficacy, behavior, and safety of nano-based applications.
\end{abstract}

Keywords: Gold nanoparticle; Dynamic flow; Interstitial fluid; Tannic acid; Cytotoxicity; Nanocellular interface 


\section{Introduction}

Due to the unique properties that arise when a material is reduced to the nano-scale, nanoparticles (NPs) are being utilized in countless applications in the public, private, and medical sectors [1]. Gold nanoparticles (AuNPs), in particular, incur a high frequency of use due to their biocompatibility, plasmonic behavior, standardized synthesis protocols, and ease of functionalization [2,3]. One primary research avenue for AuNPs are biologically-based applications, including diagnostics, imaging, photothermal therapy, and drug delivery [4]. While AuNPs hold tremendous potential, one facet that has yet to be fully understood is the nano-bio interface, which involves the dynamic interactions that exist between NPs and their cellular surroundings [5]. This complex interface is regulated by compositional, thermodynamic, and kinetic influences making prediction of its configuration and behavior exceptionally challenging. However, as the interactions between NPs and their environment regulate cellular responses, characterization of the nano-bio interface is essential for the development of nanoscience applications and methodologies.

When NPs pass into a biological environment, of both an extracellular fluid and intracellular region, they encounter local biomolecules, such as proteins, sugars, salts, and lipids. These molecules instantaneously adsorb onto the NPs' surface creating a coating around the particles, referred to as the protein corona [6]. This protein corona is dynamic by nature with its formulation depending on environmental variables, including biomolecule composition, plasma gradients, kinetic equilibrium constants, and circulation time [7,8]. Similarly, NP physicochemical properties, such as primary particle size, surface chemistry, and core composition, influence corona behavior by modulating the surface free energies and protein affinities $[9,10]$. Recent investigations have identified that the protein corona determines both the biological identify of the NPs and the cellular interactions following exposure [11,12]. In particular, the corona structure mediates NP deposition efficiency, membrane exchanges, mechanism of endocytosis, and stress activation, as it serves as a buffer between the environment and the NP core [13-16]. Therefore, the protein corona has emerged as a predominate factor in the safety, final NP biodistribution, and efficacy of nano-based applications and therapeutics. 
While significant progress has been made on elucidating corona function, one considerable drawback is that the majority of these studies have occurred either acellularly or in an in vitro environment, neither of which accurately represent the complexity of an in vivo system [16]. While the corona formation in blood and plasma have been thoroughly investigated [11,17], there exist other physiological mediums that NPs will encounter as they migrate through a body; such as interstitial and lysosomal fluids. As it is established that the protein corona varies with environment $[8,18]$, corona structure and function will likely be altered by these biological fluids. For instance, it has been shown that the protein corona is retained following cellular internalization, but becomes rapidly degraded once within lysosomes [19], highlighting the impact of biological environment. Moreover, due to the cardiovascular system, in vivo models are circulatory with dynamic flow, introducing shear stress to the NPs and providing a continual source of new biomolecules [20]. As the soft corona is comprised of loosely bound proteins, the shear force imparted could alter the size or composition of the NP-corona complex. For example, a recent study identified that multicomponent lipsosomes that were exposed to the same protein source in a dynamic, circulatory manner had a significantly modified corona composition versus static exposure [21]. However, the ability of key environmental factors, such as fluid composition and flow, to influence corona makeup and stability have yet to be satisfactorily explored [16].

As such, the goal of this investigation was to determine if, and to what extent, the physiologically relevant variables of dynamic flow and biological fluid influenced the protein corona and dependent NP characteristics. Corona evaluation was carried out with $13 \mathrm{~nm}$ AuNPs functionalized with either polyethylene glycol (PEG) or tannic acid (TA), to further elucidate the role of NP surface chemistry. Following the formation of a corona, the influences of both shear stress and fluid environment on the corona-NP complex were evaluated. Artificial biological fluids have previously been utilized to explore NP behavior and were chosen for this study due to their ability to mimic an in vivo environment while allowing for accurate and reproducible characterization of NP response and the surrounding corona [22-24]. We identified that exposure to these target physiological variables disrupted the corona structure and altered target NP parameters. Lastly, it was seen that when AuNP exposure occurred in interstitial fluid, modification to the nano-cellular interface and final dosimetry resulted in a previously unseen 
cytotoxic response within a keratinocyte cell model. Taken together, these results demonstrated that under the influence of biologically accurate variables, the protein corona-NP complex undergoes significant physical and behavioral modifications, a circumstance that needs to be considered during the design, evaluation, and implementation of nano-based applications.

\section{Materials and Methods}

\subsection{Nanoparticle Synthesis and Functionalization:}

AuNPs were synthesized using the Turkevich method, a single-step thermal reduction of chloroauric acid $\left(\mathrm{HAuCl}_{4}\right)$ in the presence of citrate [25]. Following synthesis, the AuNPs were functionalized with either thiolated-PEG or TA. The AuNP solution was centrifuged at 10,000 rcf for 10 minutes, resuspended in a $1 \mathrm{mM}$ solution of either PEG or TA, and incubated at $4{ }^{\circ} \mathrm{C}$ overnight. To ensure full functionalization, this overnight incubation process was repeated.

\subsection{Nanoparticle Characterization:}

Following synthesis, purification, and functionalization the AuNPs underwent characterization analyses to verify uniformity and to identify their unique physicochemical properties. Primary size and morphology were confirmed through transmission electron microscopy (TEM) on a Hitachi H-7600. The degree of NP agglomeration was evaluated through dynamic light scattering (DLS) on a Malvern Zetasizer Nano-ZS. The surface charge of the particles in solution was measured using a zeta potential analysis, also carried out on the Malvern Zetasizer. UVVIS analysis was used to determine alterations in the spectral signature of the AuNPs, on a Varian Cary 5000. Following AuNP incubation in the denoted environments, the particles were re-characterized through TEM, UV-VIS, and DLS to identify changes in physical properties. All characterization procedures were carried out at a concentration of $25 \mu \mathrm{g} / \mathrm{mL}$.

\subsection{Corona Isolation and SDS-PAGE Analysis:}

The AuNPs were suspended in cell culture media (RPMI 1640 base medium supplemented with $1 \%$ penicillin/streptomycin and $10 \%$ fetal bovine serum (FBS)) at a concentration of $25 \mu \mathrm{g} / \mathrm{mL}$. As FBS served as the protein source for this study, the same FBS supply was used throughout experimentation for consistency. After a 24 hour incubation at the denoted conditions, the NPs 
were pelleted via centrifugation at $10,000 \mathrm{rcf}$ for 10 minutes, washed two times to remove unattached proteins, and resuspended in $20 \mu \mathrm{L}$ phosphate buffered saline (PBS).

The bound proteins were removed from the NP surface by boiling for 5 minutes in a $2 x$ Laemmli sample buffer, using DTT with a 1x concentration of $50 \mathrm{mM}$ for the reducing agent. The NPs were removed via centrifugation and the proteins were separated through SDS-PAGE on a miniPROTEAN system (Biorad). The samples were loaded onto a 4-20\% gradient mini-PROTEAN TGX gel (Biorad) with a Precision Plus Protein Kaleidoscope ladder, and run with a $25 \mathrm{mM}$ Tris/192 mM glycine running buffer. Following electrophoresis, the gels were rinsed with deionized water and incubated with EZ Blue Gel Staining Reagent (Sigma Aldrich) for 1 hour.

\subsection{Protein Concentration Analysis:}

The protein concentration within the corona-NP complex was evaluated to verify SDS-PAGE results. The unbound proteins were removed via washing as outlined above, then the bound proteins were removed from the NP surface by boiling in a 2x Laemmli sample buffer for 5 minutes, with the NPs removed through centrifugation. The protein levels within the supernatant were analyzed through the DC Protein Assay (Biorad), in accordance with the manufacturer's instructions.

\subsection{Dynamic Flow Environment:}

A dynamic system was established through the use of a FH100M multichannel peristaltic pump (Fisher Scientific) operated with $0.8 \mathrm{~mm}$ (inner diameter) Pharmed BPT tubing. The volumetric flow rate was equal to $2.5 \times 10^{-3} \mathrm{~cm}^{3} / \mathrm{s}$, which with the $0.8 \mathrm{~mm}$ tubing produced a target linear velocity of $0.5 \mathrm{~cm} / \mathrm{s}$ within the tubing (Supporting information). This flow rate was specifically targeted due to the fact that standard physiological velocities, including those for arteries, arterioles, capillaries, and venules, range between 0.2 and $1.0 \mathrm{~cm} / \mathrm{s}$ [20]. To generate flow in a 24-well cell culture dish the tubing ends were inserted and secured through precisely drilled holes in the plate lid, as previously performed [26]. For dynamic evaluation, the AuNPs were resuspended in media at a concentration of $25 \mu \mathrm{g} / \mathrm{mL}$ then allowed to reside in the flowing system for 24 hours at $37{ }^{\circ} \mathrm{C}$. Identical batches were simultaneously evaluated for static 
conditions to allow for direct comparison. NP and corona characterization was then performed as previously described.

\subsection{Corona Evaluation in Physiological Fluids:}

To mimic an in vivo environment, artificial interstitial and lysosomal fluids were prepared as previously published [27], with $\mathrm{pH}$ values of 7.4 and 4.5, respectively. As the artificial fluids contained no proteins, a corona was created around the AuNPs prior to dispersion. To achieve this, $250 \mu \mathrm{g}$ of the AuNPs were incubated in $500 \mu \mathrm{L}$ of pure FBS for two hours at $37{ }^{\circ} \mathrm{C}$. These samples were then diluted with artificial fluid to a total volume of $10 \mathrm{~mL}$. The particles were incubated for 24 hours at $37^{\circ} \mathrm{C}$, then characterized and processed for SDS-PAGE as previously described.

\subsection{Evaluation of Cellular Behavior}

The keratinocyte cell line, HaCaT, was maintained in RPMI 1640 supplemented with 1\% penicillin/streptomycin and 10\% FBS and stored in a humidified incubator at $37{ }^{\circ} \mathrm{C}$. For viability assessments, HaCaTs were seeded in a 96-well plate at a density of $2 \times 10^{4}$ per well and incubated overnight. The cells were then exposed to the denoted fluid/AuNP combination for 24 hours, after which viability was assessed using the CellTiter 96 AQueous one solution cell proliferation assay (Promega). HaCaTs that remained untreated and incubated in media served as the negative control.

For visualization of the nano-cellular interface, HaCaTs were seeded on a two-chambered slide at a concentration of $2 \times 10^{5}$ per chamber and returned to the incubator overnight. The following day, the cells were exposed to the AuNPs in either media or interstitial fluid at a concentration of $25 \mu \mathrm{g} / \mathrm{mL}$ for 24 hours. The HaCaTs were then washed, fixed with $4 \%$ paraformaldehyde and stained with DAPI and Alexa Fluor 555-phalloidin (Invitrogen) for nuclear and actin detection, respectively. The slides were then removed, sealed, and imaged on an Olympus BX41 microscope with a CytoViva ultraresolution attachment (Aetos Technologies).

\subsection{AuNP Deposition Analysis}


$\mathrm{HaCaT}$ cells were seeded in a six-well plate at a density of $8 \times 10^{5}$ cells/well and incubated for an additional 24 hours. Cells were then dosed with the AuNPs at a concentration of $25 \mu \mathrm{g} / \mathrm{mL}$ for a 24 hour duration then washed, detached with trypsin, counted, and digested with an aqueous solution of $0.05 \%$ Triton $\mathrm{X}-100,3 \% \mathrm{HCl}$, and $1 \% \mathrm{HNO}$. The amount of intracellular gold was then quantified through ICP-MS on a Perkin Elmer NexION 300D. Deposition efficiency was determined by comparison against the total administered AuNP dosage.

\subsection{Statistical Analysis:}

All data are expressed as the mean \pm the standard error of the mean (SEM). All analyses were carried out in triplicate with three independent trials. Either a one or two way ANOVA with a Bonferroni post-test was run using Graph Pad Prism to determine statistical significance from the stated controls. Statistical significance corresponds to a p-value less than 0.05 .

\section{Results}

\subsection{Initial Nanoparticle Characterization:}

Prior to protein exposure, the stock AuNPS were characterized to determine key physicochemical properties. A large batch of colloidal gold was synthesized, equally divided, and functionalized with either PEG for stability [28] or TA for greater protein affinity [29]. Through TEM imaging the spherical morphology and primary size of approximately $13 \mathrm{~nm}$ was confirmed for both particle sets (Figure 1; Table 1).

Through DLS, it was confirmed that both particle sets agglomerated in water; with TA-AuNPs agglomerating to a larger degree, due to its well documented self-binding tendency [29]. Zeta potential analysis identified a negative surface charge for both particle sets in water (Table 1). However, the surface charge of TA AuNPs was more negative, in agreement with previous literature [29]. Lastly, the spectral signature of the particles (Figure 1C) indicated a slight rightshift and peak broadening with TA coating. These spectral alterations occur as a result of NP agglomeration [30], which is in agreement with the DLS data. Taken together, the spectral profiles, degree of agglomeration, and surface charge results confirm differential AuNP behavior induced through alterations in surface functionalization.

\subsection{Influence of Dynamic Flow:}


Following initial characterization, we sought to explore how physiological factors influenced the protein corona and subsequent NP behavior. The first variable examined was a dynamic environment. For both particle sets, static incubation in media induced a right-shift in the spectral signature compared to the spectra in pure water (Figure 2A). This shift is indicative of NP agglomeration that typically occurs in media due to the high ionic strength [30]. Agglomeration was confirmed through DLS analysis, which identified a substantial increase in agglomerate size in media versus water environments (Figure 2B).

The flow rate of the system was specifically chosen to produce an average linear velocity within the tubes of $0.5 \mathrm{~cm} / \mathrm{s}$, which is equivalent to standard flow rates within the cardiovascular system [20]. For both TA- and PEG-AuNPs, no significant modifications to the spectral profile or agglomeration were identified between static and dynamic conditions (Figures 2A and 2B); as assessed through UV-VIS and DLS analyses. These characterization results are not surprising given the laminar flow regime that the AuNPs were exposed to (Supporting Information).

However, SDS-PAGE analysis demonstrated that dynamic flow changed the protein corona structure, with dynamic samples displaying increased band intensity and number (Figure 2C). These results suggest that circulating AuNPs adsorbed a greater number of proteins, due to increased NP-protein interactions. This finding is in agreement with recent literature that identified altered corona composition following particle movement [21]. Through inspection, it appears that the protein levels associated with TA AuNPs are slightly greater than the PEG coating, agreeing with the known protein affinity of TA.

To verify the SDS-PAGE results, protein concentrations within the corona-NP complex were evaluated (Figure 2D). Firstly, these results confirmed that regardless of surface chemistry, the protein content associated with dynamic flow was increased by approximately $25 \%$ over static conditions. Protein concentrations also indicated that TA coronas were roughly $10 \%$ larger, indicating that surface chemistry does influence final makeup of the NP-corona complex. These results were further verified by converting SDS-PAGE images into binary form and quantifying pixel density using Image J software (Supporting Figure 1); which strongly correlated to protein concentrations. Taken together these results indicate that while NP characteristics remain 
unaltered under the influence of physiologically relevant flow rates, the protein corona was subject to modification.

\subsection{Influence of Environmental Composition:}

Next, we assessed the influence of fluid composition on both NP characteristics and the coronaNP complex. Circulating NPs have a strong likelihood of encountering multiple physiological environments, such as interstitial and lysosomal fluids; of which the influence on the corona have been minimally explored. Interstitial fluid is one of the most prevalent in vivo environments, as it comprises the fluid surrounding cells within tissues and organs. Following cellular internalization via endocytosis, NPs encounter lysosomal fluid, which due to its low $\mathrm{pH}$ has great potential for serious and irreversible modification to particle and corona characteristics [31]. For this study, AuNPs were first incubated in FBS to generate a protein corona, as both the synthesized interstitial and lysosomal fluids contain no proteins. The particles were then incubated in media, interstitial fluid, or lysosomal fluid, followed by characterization of AuNP properties and the associated protein corona (Figures 3-5).

Through TEM imaging, it was seen that incubation in physiological fluids induced extensive particle fusing versus media exposure (Figure 3). Furthermore, fluid-dependent shifts in the spectral signature were detected for both TA- and PEG-AuNPs (Figure 4), with PEG coating associated with a more pronounced effect. Independent of surface chemistry, exposure to artificial interstitial and lysosomal fluids resulted in a substantial increase in agglomerate size. Dispersion in interstitial fluid resulted in the formation of aggregates of approximately $300 \mathrm{~nm}$ in size (Figures 4B and 4D). These spectral shifts and increased agglomerate size are in agreement with the TEM images that demonstrate particle fusing following exposure to biological fluids.

Next, the influence of environmental composition on the NP-protein corona complex was evaluated using SDS-PAGE analysis (Figure 5A). Through direct comparison to media incubation, it was seen that exposure to both interstitial and lysosomal fluid resulted in a loss of protein, as witnessed by smaller bands. This decrease was more significant with lysosomal fluid, due to its acidic $\mathrm{pH}$. For lysosomal fluid, decrease in protein content could arise due to either 
detachment from the AuNP surface or degradation. For interstitial exposure, loss of the protein is attributed to diffusion into the surrounding environment.

To verify the loss of proteins in physiological fluids, the protein concentration within the coronaNP complex was determined for all experimental conditions (Figure 5B). For both PEG- and TA-AuNPs, the protein concentration was reduced by approximately $20 \%$ in interstitial fluid and $30 \%$ in lysosomal fluid; supporting SDS-PAGE results. Protein concentrations again determined a higher protein content associated with TA-AuNPs over a PEG surface chemistry. These results were further verified through Image $\mathbf{J}$ analysis of pixel density (Supporting Figure 2).

\subsection{Modified Nano-Cellular Interface as a Function of Environmental Composition:}

In this study, we established that both AuNP behavior and corona stability were modified following exposure to interstitial fluid. As the corona has been shown to direct NP-cellular interactions, it stands to reason that within an interstitial environment, the nano-cellular interface would be reshaped. Therefore, the next goal of this study was to ascertain if the fluid-induced alterations to the protein-NP complex extended to modified cellular responses. A keratinocyte cell model, HaCaT, was selected due to the considerations that interstitial fluid is the predominant environment of skin tissue and that the $\mathrm{HaCaT}$ line has become a model for NP investigations $[32,33]$.

First, the nano-cellular interface was visualized through high-resolution fluorescence microscopy (Figure 6). In interstitial fluid large AuNP agglomerates were easily identified, in agreement with the previous DLS and TEM data. This effect is especially pronounced with PEG-AuNPs, which agglomerate to a lesser degree in media. Therefore, in addition to disrupting the protein corona institial fluid exposure altered the way a cellular system encounters and interacts with AuNPs. Supporting Information Figure 3 includes images of $\mathrm{HaCaT}$ cells in media or interstitial fluid, without AuNPs, to verify that exposure to interstitial fluid did not introduce morphological variations.

The images of the nano-cellular interface suggested that the AuNP dosimetry varied as a function

of both surface chemistry and fluid environment. As final delivered dose strongly correlates to 
observed biological responses [34,35], we next sought to quantify the AuNP deposition (Figure 7A). Results identified that in media, TA-AuNPs deposited on the HaCaT surface to a higher degree than PEG. However, in interstitial fluid, both sets of AuNPs were deposited to a higher degree, likely due to increased particle agglomeration and sedimentation [25,34].

As both the protein corona and nano-cellular interactions have been shown to dictate the NPinduced biological response, we next evaluated if AuNP exposure within interstitial fluid elicited unique bioeffects. To assess this, a standard viability metric was selected and performed on HaCaT cells exposed to AuNPs in either media or interstitial fluid (Figure 7B). In media, exposure to a $25 \mu \mathrm{g} / \mathrm{mL}$ concentration of PEG- or TA- AuNPs induced a negligible cytotoxic response, in agreement with their established biocompatibility [36]. When HaCaTs were exposed to interstitial fluid alone or jointly with PEG-AuNPs, no cell death was noted. However, during joint exposure with TA-AuNPs and interstitial fluid, a combinatorial effect occurred that resulted in a $32 \%$ loss of HaCaT viability. As PEG-AuNPs displayed no negative response, our data indicates that this unique bioeffect is dependent on both environment and particle surface chemistry.

\section{Discussion}

From both this work and other previous reports, it is known that the protein corona-NP complex is a dynamic entity, dependent on a number of environmental and physiochemical properties $[8,9]$. It is known that NP surface chemistry is a major factor in subsequent behavior and corona formation, and was therefore selected as a focus of this study [37]. The main goal of this study was to identify if, and to what extent, physiological variables could disrupt the protein corona around AuNPs and modify target physicochemical properties. As the protein corona dictates NP response in a biological setting, generating a solid foundation of knowledge regarding to how key physiological variables affect this response is necessary for the development of nano-based therapeutics and applications [11,12].

Under dynamic flow, we identified that the protein corona was modified through the adsorption and binding of different proteins (Figure 2). These results are in agreement with a previous study that identified that following incubation in a circulatory system the protein corona contained a 
larger number of apolipoproteins and acute phase proteins than static conditions [16]. This phenomena is likely due to the combination of continual protein encounters and reduced particle sedimentation brought on by the presence of fluid flow [34]. Using a physiologically relevant linear velocity of $0.5 \mathrm{~cm} / \mathrm{s}$ and standard shear stress models for laminar flow through a tube, the shear stress imparted by the flow was determined to be approximately 0.4 dyne $/ \mathrm{cm}^{2}$ (Supporting information). Standard shear values within the cardiovascular system, which are approximately 6-10 dyne $/ \mathrm{cm}^{2}$, are greater due to the small vasculature diameter and are currently unobtainable while maintaining the target velocity of $0.5 \mathrm{~cm} / \mathrm{s}$ with standard size tubing [20]. Therefore, these values indicate that when in an actual in vivo environment, shear stress appears will be greater and could potentially cause greater corona remodeling not observed in this study. While this study identified that the corona is subject to modification from dynamic flow, further investigation is required to full elucidate the mechanism behind these alterations.

Modification to the protein corona and NP properties were observed following incubation in interstitial and lysosomal fluids (Figures 3-5). Interstitial fluid is the most prevalent fluid in an in vivo system and is known to impact NP characteristics [22,23], but its influence on the protein corona had been previously unexplored. As artificial interstitial fluid had no protein component, a significant concentration gradient existed between the corona and the environment: providing a driving force for protein dissociation. This driving force provides a probable mechanism for the decrease in protein concentration in interstitial fluid. Protein loss was even greater following dispersion in artificial lysosomal fluid, which we believe arises from partial protein degradation due to the acidic $\mathrm{pH}$. As real lysosomal fluid possess a great endocytotic capability, which target and degrade proteins, we believe that corona disruption will be even greater in vivo [38]. This supposition, combined with our results of corona disruption, highlight the necessity of evaluation NP-corona behavior in multiple physiologically relevant environments.

Even without a significant protein content, $\mathrm{HaCaT}$ exposure to AuNPs while in an interstitial fluid environment was successfully achieved and examined. While cellular proliferation would have been altered, our results demonstrated that for short time periods the cells were capable of surviving with this environment. Most importantly, changes in the nano-cellular interface were observed, with deposition patterns dependent upon the surrounding environment. In interstitial 
fluid, a distinctive shift from individual particles to large agglomerates was identified (Figure 6) and supported by quantification of AuNP deposition efficiency (Figure 7A). In the case of TAAuNPs, but not PEG coated, a synergistic cytotoxic response occurred (Figure 7). This finding correlated with observed loss of protein corona integrity (Figure 5); keeping in mind that TAAuNPs agglomerated to a larger degree, bound more protein, and had a greater deposited concentration. Taken together, these results suggest that when in a physiological environment, the corona-TA-AuNP complex is disrupted, which when combined with the presence of large agglomerates modified both the nano-cellular interface and NP dosimetry; creating a unique circumstance and potential to induce previously unseen biological consequences.

\section{Conclusions}

The protein corona surrounding NPs has recently emerged as a target and mediator of the nanocellular interface and NP behavior patterns. Numerous studies have identified that the corona is highly dynamic, with its formation and structure dependent on both NP characteristics and environmental variables. In the present report, we established that both AuNP behavior and protein corona composition were substantially altered following the introduction of key physiological factors. Under biologically accurate dynamic flow, regardless of AuNP surface chemistry, the size of the protein corona increased, as assessed through SDS PAGE and concentration analyses. Through the use of artificial fluids, it was identified that when dispersed in physiologically relevant environments, a shift in NP and corona structure occurred. Lastly, when a cellular system was jointly exposed to TA-AuNPs and interstitial fluid a synergistic cytotoxic response occurred, presumably due to the degradation of the protein corona, subsequent modification to the nano-cellular interface, and altered dosimetry. These findings highlight the need to include physiological variables during the characterization and evaluation of NPs and nano-based application in order to accurately elucidate their behavior, safety, and stability.

\section{Acknowledgments}

The authors would like to thank Ms. Elizabeth Maurer for assistance with TEM imaging. N.J.B. was supported through the Repperger Research Intern Program funded through the Air Force

Chief Scientist's Office. M.C.D. was supported through the Oak Ridge Institute for Science and 
Education (ORISE). K.K.C. was jointly funded by the Summer Faculty Fellowship Program supported through the Air Force Office of Scientific Research and through the University of Dayton Research Council Seed Grant.

\section{REFERENCES}

[1] X. Zhang, Gold nanoparticles: recent advances in the biomedical applications, Cell Biochem. Biophys. 72 (2015) 771-775.

[2] Y.C. Yeh, B. Creran, V.M. Rotello, Gold nanoparticles: preparation, properties, and applications in bionanotechnology. Nanoscale 4 (2012) 1871-1880.

[3] B.M. Stacy, K.K. Comfort, D.A. Comfort, S.M. Hussain, In vitro identification of gold nanorods through hyperspectral imaging. Plasmonics 8 (2013) 1235-1240.

[4] L. Dykman, N. Khlebtsov, Gold nanoparticles in biomedical applications: recent advances and perspectives. Chem. Soc. Rev. 41 (2012) 2256-2282.

[5] X. Liu, S. Wang, Three-dimensional nano-biointerface as a platform for guiding cell fate. Chem. Soc. Rev. 43 (2014) 2385-2401.

[6] M. Lundqvist, J. Stigler, T. Cedervall, T. Berggard, M.B. Flanagan, I. Lynch, G. Elia, K. Dawson, The evolution of the protein corona around nanoparticles: a test study. ACS Nano 5 (2011) 7503-7509.

[7] A.L. Barran-Berdon, D. Pozzi, G. Caracciolo, A.L. Capriotti, G. Caruso, C. Cavaliere, A. Riccioli, S. Palchetti, A. Lagana, Time evolution of nanoparticle-protein corona in human plasma: relevance for targeted drug delivery. Langmuir 29 (2013) 6485-6494.

[8] M. Ghavami, S. Saffar, B. Emamy, A. Peirovi, M.A. Shokrgozar, V. Serpooshan, M. Mahmoudi, Plasma concentration gradient influences protein corona decoration on nanoparticles. RSC Adv. 3 (2013) 1119-1126.

[9] M.M. Yallapu, N. Chauhan, S.F. Othman, V. Khalizad-Sharghi, M.C. Ebeling, S. Khan, M. Jaggi,S.C. Chauhan, Implications of the protein corona on physico-chemical and biological properties of magnetic nanoparticles. Biomaterials 46 (2015) 1-12.

[10] L. Shang, K. Nienhaus, G.U. Nienhaus, Engineered nanoparticles interacting with cells: size matters. J. Nanobiotechnol. 12 (2014) 5.

[11] M.P. Monopoli, C. Aberg, A. Salvati, K.A. Dawson, Biomolecular coronas provide the biological identify of nanosized materials. Nat. Nanotechnol. 7 (2012) 779-786. 
[12] P. Foroozandeh, A.A. Aziz, Merging worlds of nanomaterials and biological environment: factors governing protein corona formation on nanoparticles and its biological consequences. Nanoscale Res. Lett. 10 (2015) 221.

[13] S. Tenzer, D. Docter, J. Kuharev, A. Musyanovych, V. Fetz, R. Hecht, F. Schlenk, D. Fischer, K. Kiouptsi, C. Reinhardt, K. Landfester, H. Schild, M. Maskos, S.K. Knauer, R.H. Stauber, Rapid formation of plasma protein corona critically affects nanoparticle pathophysiology. Nat. Nanotechnol. 8 (2013) 772-781.

[14] C. Gunawan, M. Lim, C.P. Marquis, R. Amal, Nanoparticle-protein corona complexes govern the biological fates and function of nanoparticles. J. Mat. Chem. B. 2 (2014) 2060-2083.

[15] A.O. Luby, E.K. Breitner, K.K. Comfort, Preliminary protein corona formation stabilizes gold nanoparticles and improves deposition efficiency. Appl. Nanosci. (2015) DOI: 10.1007/s13204-015-0501-z.

[16] M. Hadjidemetriou, Z. Al-Ahmady, M. Mazza, R.F. Collins, K. Dawson, K. Kostarelos, In vivo biomolecule corona around blood-circulating, clinically used and antibody-targeted lipid bilayer nanoscale vesicles. ACS Nano 9 (2015) 8142-8156.

[17] C.D. Walkey, W.C. Chan, Understanding and controlling the interaction of nanomaterials with protein in a physiological environment. Chem. Soc. Rev. 41 (2012) 2780-2799.

[18] M.P. Monopoli, D. Walczyk, A. Campbell, G. Elia, I. Lynch, F.B. Bombelli, K.A. Dawson, Physical-chemical aspects of protein corona: relevance to in vitro and in vivo biological impacts of nanoparticles. J. Am. Chem. Soc. 133 (2011) 2525-2534.

[19] F. Wang, L. Yu, M.P. Monopoli, P. Sandin, E. Mahon, A. Salvati, K.A. Dawson, K.A. The biomolecular corona is retained during nanoparticle uptake and protects the cells from the damage induced by cationic nanoparticles until degraded in the lysosomes. Nanomedicine 9 (2013) 1159-1168.

[20] S.A. Berger, W. Goldsmith, E.R. Lewis, Introduction to bioengineering. Oxford: Oxford University Press, 1996.

[21] D. Pozzi, G. Caracciolo, L. Digiacomo, V. Colapichioni, S. Palchetti, A.L. Capriotti, C. Cavaliere, R.Z. Chiozzi, A. Puglisi, A. Lagana, The biomolecular corona of nanoparticles in circulating biological media. Nanoscale 7 (2015) 13958-13966. 
[22] K.K. Comfort, J.W. Speltz, B.M. Stacy, L.R. Dosser, S.M. Hussain, Physiological fluid specific agglomeration patterns diminish gold nanorod photothermal characteristics. Adv. Nanopart. 2 (2013) 336-343.

[23] L.K. Braydich-Stolle, E.K. Breitner, K.K. Comfort, J.J. Schlager, S.M. Hussain, Dynamic characteristics of silver nanoparticles in physiological fluids: toxicological implications. Langmuir 30 (2014) 15309-15316.

[24] A. Fashina, E. Antunes, T. Nyokong, Silica nanoparticles grafted with phthalocyanines: photophysical properties and studies in artificial lysosomal fluid. New J. Chem. 37 (2013) 28002809.

[25] J. Tukevich, P.C. Stevenson, J. Hillier, A study of the nucleation and growth processes in the synthesis of colloidal gold. Discuss. Faraday Soc. 11 (1951) 55-75.

[26] E.K. Breitner, S.M. Hussain, K.K. Comfort, The role of biological fluid and dynamic flow in the behavior and cellular interactions of gold nanoparticles. J. Nanobiotechnol. 13 (2015) 56.

[27] W. Stopford, J. Turner, D. Cappellini, T. Brock, Bioaccessibility testing of cobalt compounds. J. Environ. Monit. 4 (2003) 675-680.

[28] G. Park, D. Seo, I.S. Chung, H. Song, Poly(ethylene glycol)- and carboxylate-functionalized gold nanoparticles using polymer linkages: single-step synthesis, high stability, and plasmonic detection of proteins. Langmuir 29 (2013) 13518-13526.

[29] E.A. Untener, K.K. Comfort, E.I. Maurer, C.M. Grabinski, D.A. Comfort, S.M. Hussain, Tannic acid coated gold nanorods demonstrate a distinctive form of endosomal uptake and unique distribution within cells. ACS Appl. Mater. Interfaces 5 (2013) 8366-8373.

[30] M. Sethi, G. Joung, M.R. Knecht, Stability and electrostatic assembly of Au nanorods for use in biological assays. Langmuir 25 (2008) 317-325.

[31] K.K. Comfort, L.K. Braydich-Stolle, E.I. Maurer, S.M. Hussain, Less is more: long-term in vitro exposure to low levels of silver nanoparticles provides new insights for nanomaterial evaluation. ACS Nano 8 (2014) 3260-3271.

[32] H. Liang, C. Jin, Y. Tang, F. Wang, C. Ma, Y. Yang, Cytotoxicity of silica nanoparticles on HaCaT cells. J. Appl. Toxicol. 34 (2014) 367-372.

[33] K.K. Comfort, E.I Maurer, S.M. Hussain, Slow release of ions from internalized silver nanoparticles modifies the epidermal growth factor signaling response. Colloids Surf. B Biointerfaces 123 (2014) 136-142. 
[34] P.M. Hinderliter, K.R. Minard, G. Orr, W.B. Chrisler, B.D. Thrall, J.G. Pounds, J.G. Teeguarden, ISDD: a computational model of particle sedimentation, diffusion and target cell dosimetry for in vitro toxicity studies. Part. Fibre Toxicol. 7 (2010) 36.

[35] M. Kang, C.H. Lim, J.H. Han, Comparison of toxicity and deposition of nano-sized carbon black aerosol prepared with or without dispersing sonication. Toxicol. Res. 29 (2013) 121-127.

[36] C.J. Murphy, A.M. Gole, J.W. Stone, P.N. Sisco, A.M. Alkilany, E.C. Goldsmith, S.C. Baxter, Gold nanoparticles in biology: beyond toxicity to cellular imaging. Acc. Chem. Res. 41 (2008) 1721-1730.

[37] D. Pozzi, G. Caracciolo, A.L. Capriotti, C. Cavaliere, G. LaBarbera, T.J. Ancordoguy, A. Lagana, Surface chemistry and serum type both determine the nanoparticle-protein corona. J. Proteomics 119 (2015) 209-217.

[38] T. Yamaza, M.A. Kido, T. Kiyoshima, Y. Nishimura, M. Himeno, T.A. Tanaka, Fluid-phase endocytotic capacity and intracellular degradation of a foreign protein (horseradish peroxidase) by lysosomal cysteine proteinases in the rat junctional epithelium. J. Periodontal. Res. 32 (1997) 651-660. 
List of Tables:

Table 1: Characterization of stock AuNPs. 


\section{Figure Legends:}

Figure 1: Characterization of the stock AuNPs. Representative TEM of the (A) PEG-AuNPs and (B) TA-AuNPs verified their spherical morphology and were used to determine primary particle size. (C) The spectral signature of the AuNPs demonstrated a plasmonic peak at approximately $540 \mathrm{~nm}$ and a coating-dependent spectral shift associated with TA.

Figure 2: Dynamic flow alters the NP-protein corona complex. (A) Following 24 hour incubation in a dynamic flow environment, AuNP characteristics demonstrated no significant change, as determined through (A) UV-VIS analysis and (B) degree of agglomeration. (C) SDSPAGE evaluation showed increased protein content for dynamic conditions. Lanes marked S and D represent static and dynamic, respectively. A constant number of AuNPs were loaded per lane for normalization. (D) The quantity of protein bound to AuNPs was quantified and supports SDS-PAGE analysis. Protein concentrations were normalized against static TA AuNPs values. A two way ANOVA was run with * denoting significance between surface chemistries and \$ denoting significance between static and dynamic for the same AuNP set $(n=3, p<0.05)$.

Figure 3: AuNP visualization in physiological fluids. Representative TEM images of the AuNPs following a 24 hour incubation in media, artificial interstitial, or artificial lysosomal environments. TA- and PEG-AuNPs are shown in images $\mathrm{A} / \mathrm{C} / \mathrm{E}$ and $\mathrm{B} / \mathrm{D} / \mathrm{F}$ respectively. These images demonstrate how exposure to physiological fluids induced changes to NP physicochemical properties.

Figure 4: Environment-specific modifications to AuNP characterization. Following the generation of a protein corona and 24 hour incubation in media, interstitial fluid, or lysosomal fluid TA-AuNP characteristics were evaluated through (A) spectral analysis and (B) identification of particle agglomeration tendencies. Images (C) and (D) include the spectral and agglomerate analyses for PEG-AuNPs, respectively. These analyses demonstrate modified AuNP behavior in physiological fluids. This figure represents three independent trials, * denotes statistical significance from media sample with $\mathrm{p}<0.05$. 
Figure 5: Environment-specific modifications to the protein corona. (A) Following a 24 hour incubation in media (M), interstitial (I), or lysosomal (L) fluids a SDS-PAGE analysis was performed to visualize the influence of environment composition on the protein corona surrounding AuNPs. A constant number of either TA or PEG coated AuNPs were loaded per lane for normalization. (B) Following incubation, the protein concentration associated with the corona-NP complex was evaluated and normalized against TA-AuNPs media conditions. A two way ANOVA was run with * denoting significance between surface chemistries and $\$$ denoting significance between fluids for the same AuNP set $(n=3, p<0.05)$.

Figure 6: The nano-cellular interface is dependent on both surface chemistry and environment. $\mathrm{HaCaT}$ cells were exposed to AuNPs in either media or interstitial fluid for 24 hours, after which the nano-cellular interface was evaluated with fluorescence microscopy. In these representative images, the cells underwent nuclear (blue) and actin (red) staining, with the AuNP appearing as white.

Figure 7: AuNP dosimetry and $\mathrm{HaCaT}$ viability are a function of surface chemistry and environmental composition. (A) The AuNP deposition efficiency was assessed after a 24 hour exposure in the denoted conditions. A two way ANOVA was run with * denoting significance between surface chemistries and $\$$ denoting significance between fluids for the same AuNP set $(\mathrm{n}=3, \mathrm{p}<0.05)$. (B) Following a 24 hour incubation in a combination of media or interstitial fluid and either PEG- or TA-AuNPs, $\mathrm{HaCaT}$ viability was assessed. These results represent three independent trials, with $*$ denotes statistical significance from media control and a $\mathrm{p}<0.05$. 

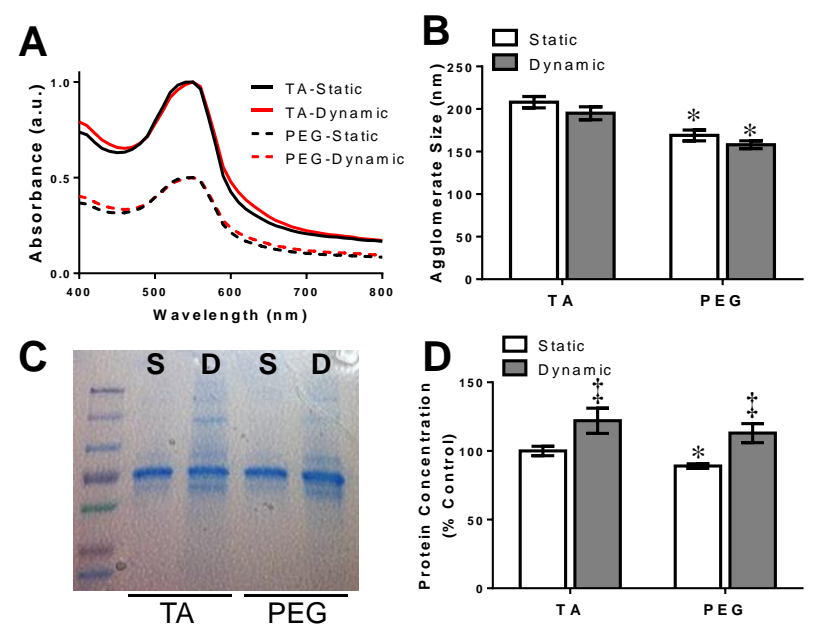


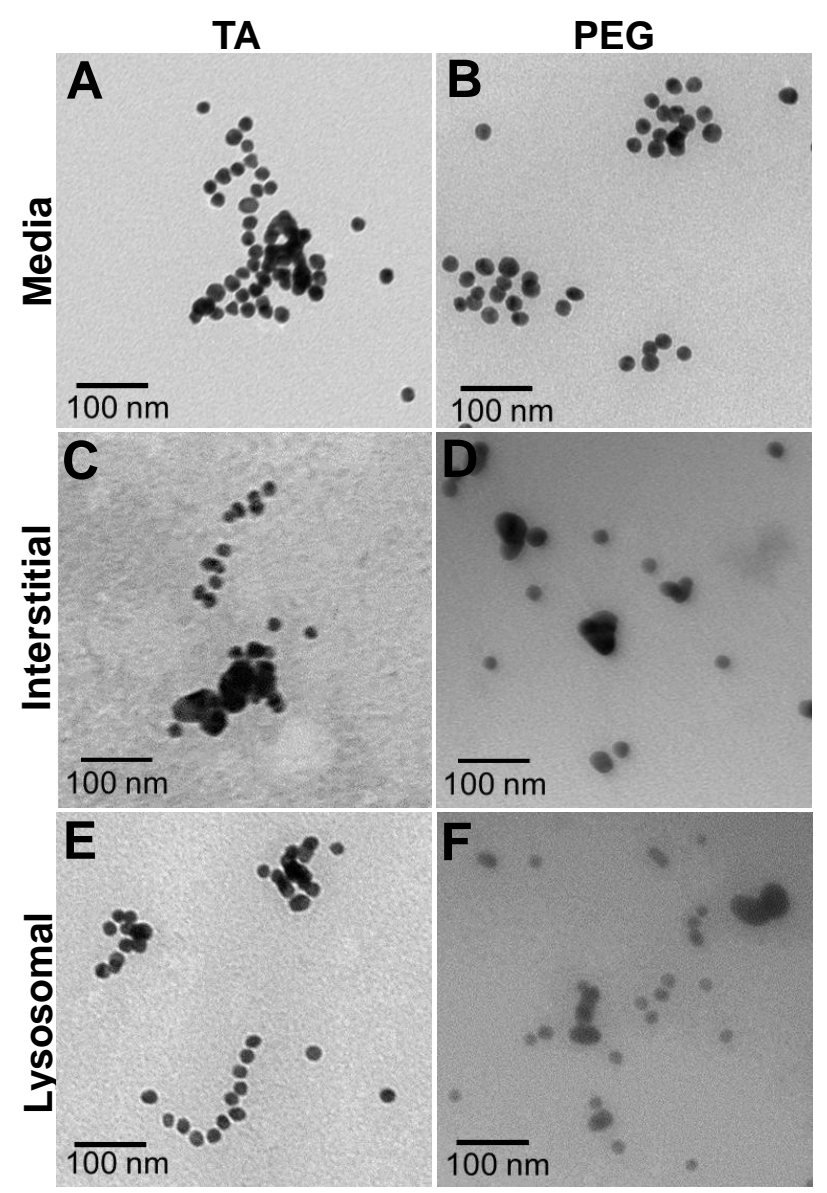



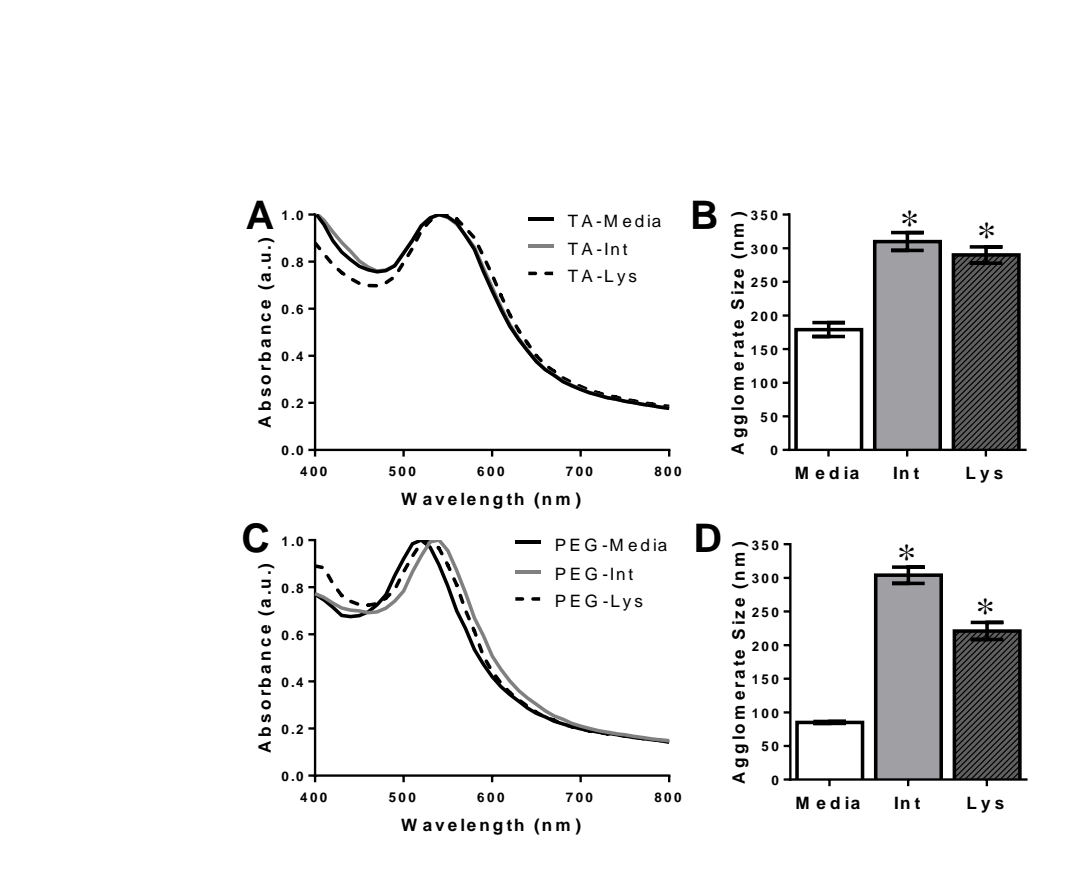

Figure 4

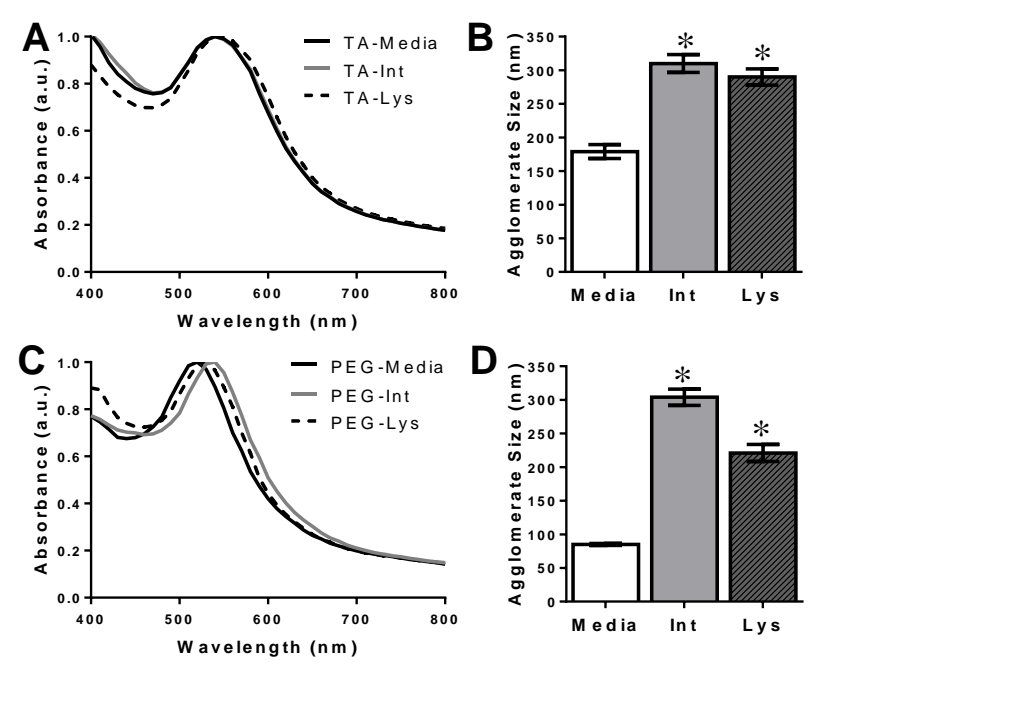

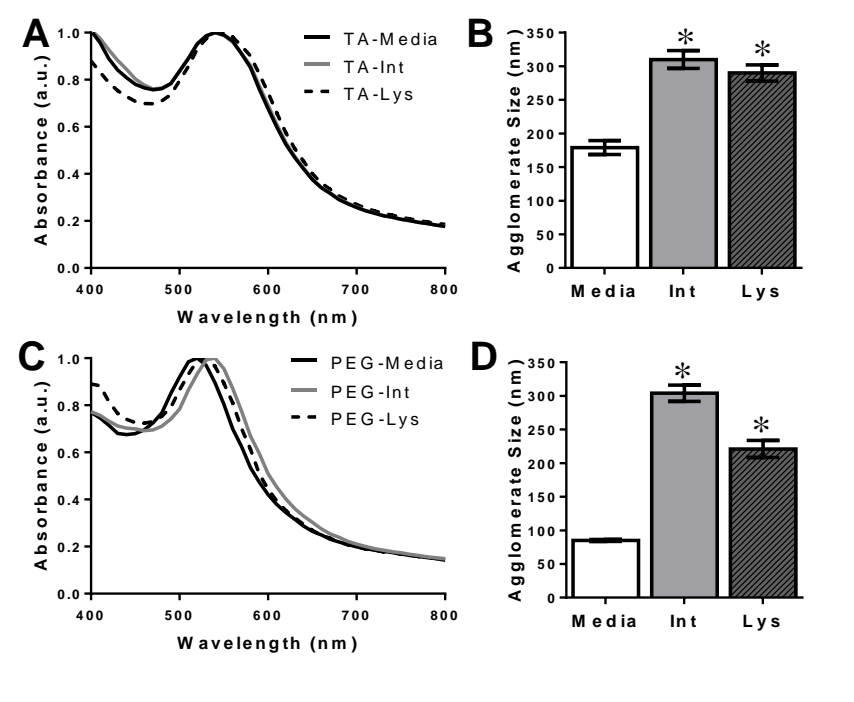

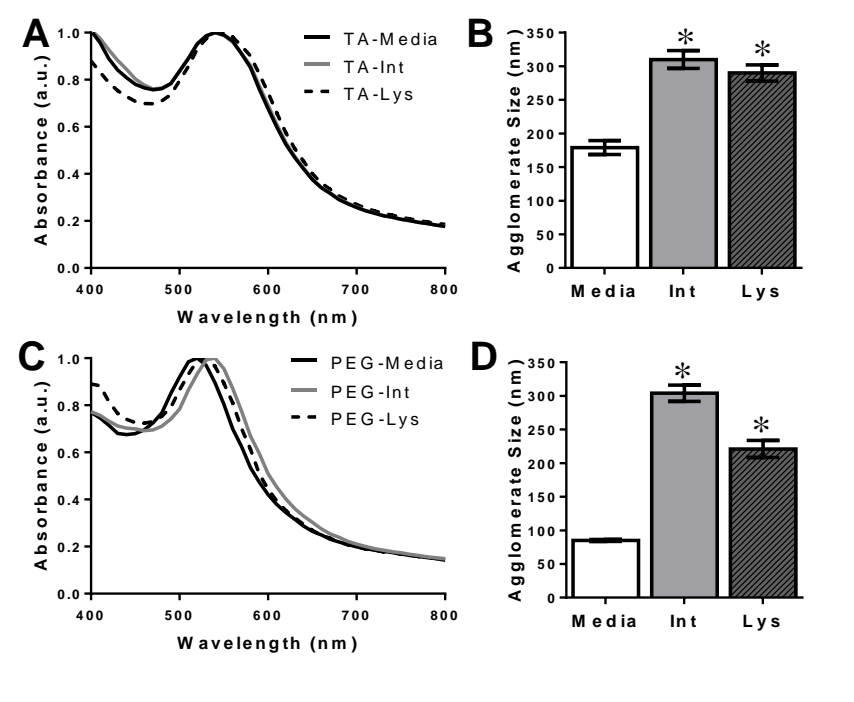

$$
\text { . }
$$
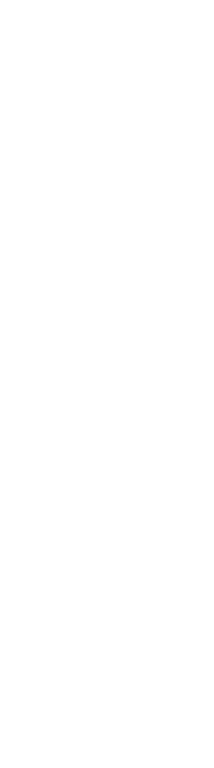

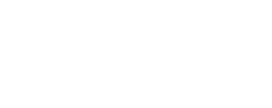

$$
\text { . }
$$

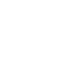




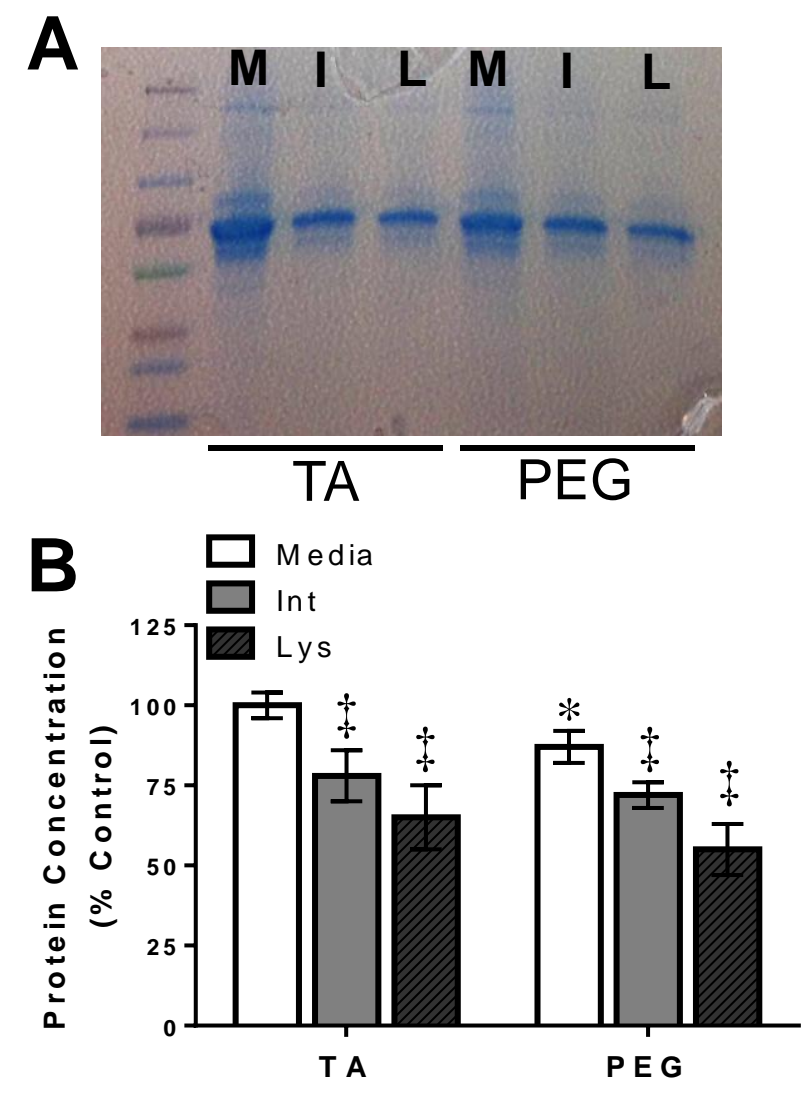




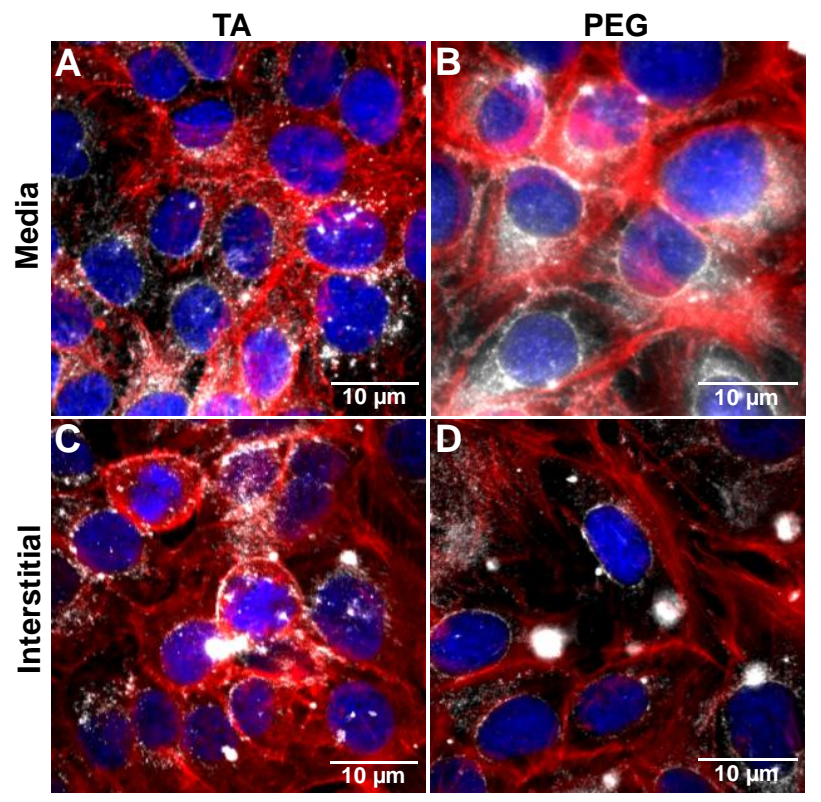

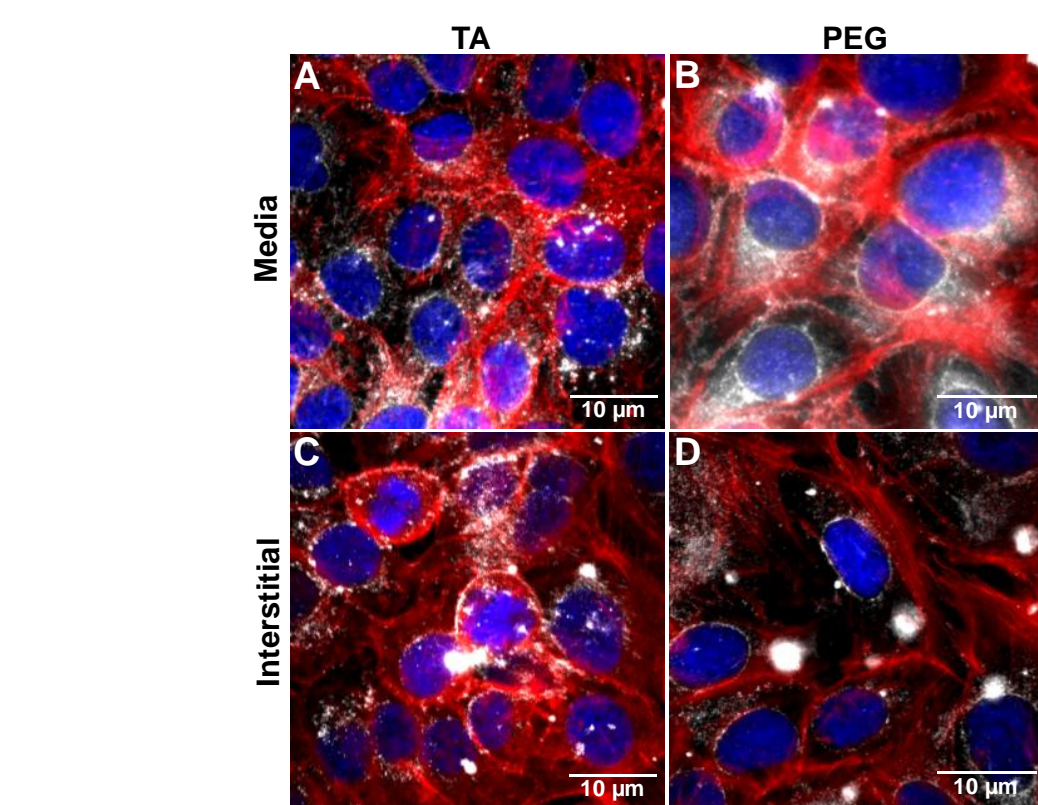
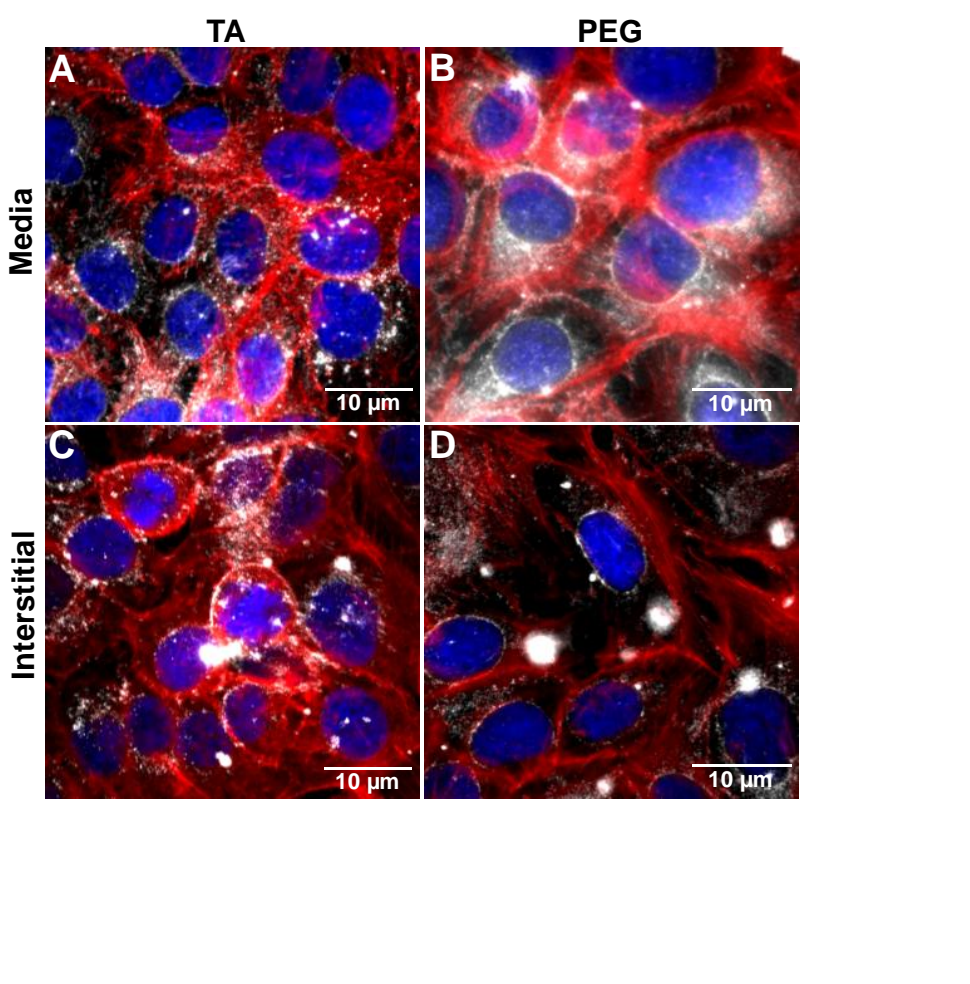

.
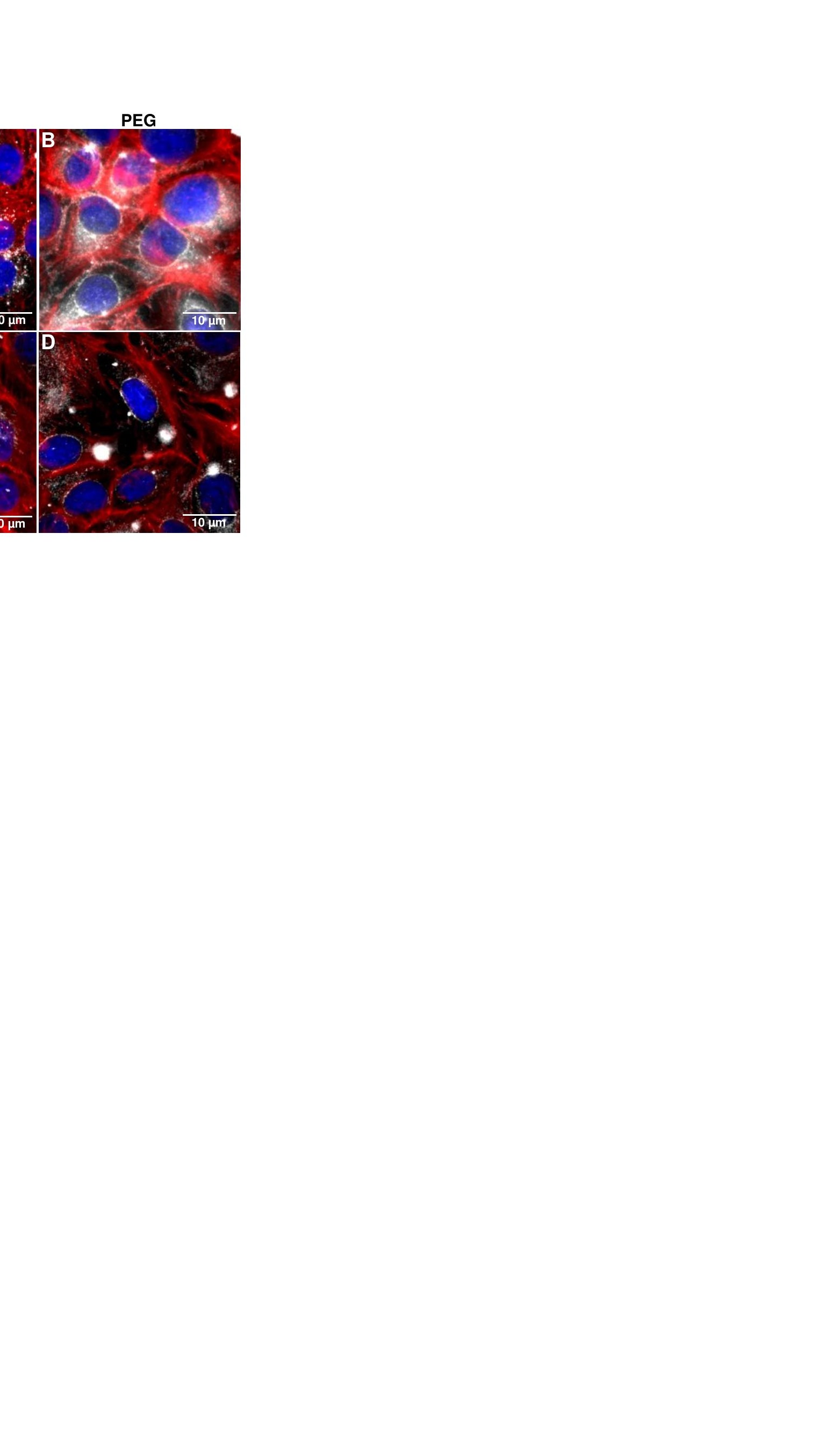


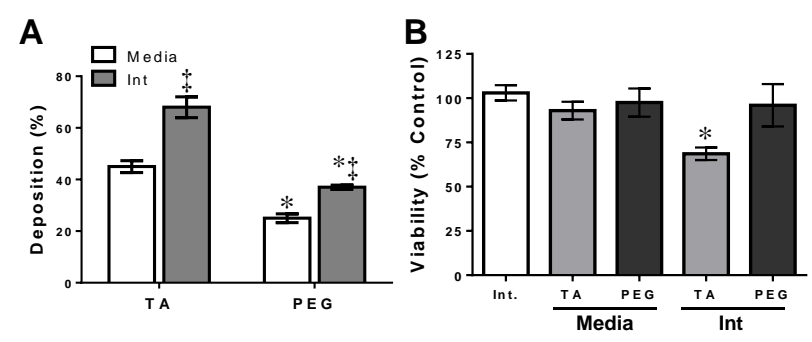

Figure 7

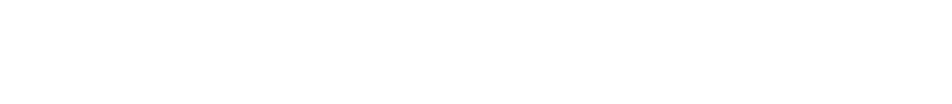




\begin{tabular}{|l|l|l|l|}
\hline & Primary Size (nm) & Agglomerate Size (nm) & Zeta Potential (mV) \\
\hline$P E G$ & $13.3 \pm 0.7$ & $85.9 \pm 3.6$ & $-13.5 \pm 0.4$ \\
\hline$T A$ & $13.2 \pm 0.8$ & $165.7 \pm 12.2$ & $-28.1 \pm 2.5$ \\
\hline
\end{tabular}

